\title{
Design and Prediction of a Novel Two-Dimensional Carbon Nanostructure with In-Plane Negative Poisson's Ratio
}

\author{
Kun Yuan $\mathbb{D}^{1},{ }^{1,2,3}$ Meng-Yang $\mathrm{Li}^{2}$ Yan-Zhi Liu, ${ }^{1}$ and Ren-Zhong $\mathrm{Li}^{3}$ \\ ${ }^{1}$ College of Chemical Engineering and Technology, Tianshui Normal University, Tianshui 741001, China \\ ${ }^{2}$ Institute for Chemical Physics \& Department of Chemistry, School of Science, State Key Laboratory of Electrical Insulation and \\ Power Equipment, School of Mechanical Engineering, Xi'an Jiaotong University, Xi'an 710049, China \\ ${ }^{3}$ School of Environmental and Chemical Engineering, Xi'an Polytechnic University, Xi'an 710049, China \\ Correspondence should be addressed to Kun Yuan; yuankun@tsnu.edu.cn
}

Received 4 November 2018; Revised 4 January 2019; Accepted 10 January 2019; Published 25 February 2019

Academic Editor: Xuping Sun

Copyright (c) 2019 Kun Yuan et al. This is an open access article distributed under the Creative Commons Attribution License, which permits unrestricted use, distribution, and reproduction in any medium, provided the original work is properly cited.

\begin{abstract}
The intrinsic negative Poisson's ratio effect in 2-dimensional nanomaterials have attracted a lot of research interests due to its superior mechanical properties, and new mechanisms have emerged in the nanoscale. In this paper, we designed a novel graphyne-like two-dimensional carbon nanostructure with a "butterfly" shape (GL-2D-1) and its configuration isomer with a "herring-bone" form (GL-2D-2) by means of density functional theoretical calculation and predicted their in-plane negative Poisson's ratio effect and other mechanical properties. Both GL-2D-1 and GL-2D-2 present a significant negative Poisson's ratio effect under different specific strains conditions. By contrast, GL-2D-2 presents a much stronger negative Poisson's ratio effect and mechanical stability than does GL-2D-1. It is hoped that this work could be a useful structural design strategy for the development of the $2 \mathrm{D}$ carbon nanostructure with the intrinsic negative Poisson's ratio.
\end{abstract}

\section{Introduction}

It is known that carbon can exist in several different structures. Besides the traditional allotropes [1], some other allotropes of carbon with nanostructures including fullerenes, carbon nanotubes, graphene, graphyne, T-carbon, and graphane were found one after another [2-12]. However, researchers in physical chemistry never stop to explore newer allotropes from zero to three dimensions $[13,14]$. As Hobza pointed out [15], "the way of covalent interactions determine $[s i c]$ the primary structure of a molecule." The diversity of the carbon allotrope derives from its three kinds of hybridization ability, namely, $s p, s p^{2}$, and $s p^{3}$ hybridizations. Two-dimensional (2D) carbon allotropes have received much attention in the last ten years due to their potential application in nanoelectronics, optoelectronics, biomaterials [16-24], energy storage [25], and gas separation [26]. But to date, the 2D carbon and analogous materials are not quite abundant. Therefore, it deserves exploration in theory and in experiment whether there exists other $2 \mathrm{D}$ carbon allotropes which may be stable as a monolayer and exhibit novel properties, especially novel mechanical properties. If confirmative, it is likely to make important contributions to the multifunctional carbon nanomaterials.

Since the first observation of the auxetic phenomenon in polyurethane foam by Lakes [27] three decades ago, great efforts have been made to find or design new auxetic materials which are also known as negative Poisson's ratio materials. Most materials will be contracted in the lateral direction when they are stretched, generally resulting in a positive value for Poisson's ratio. Abnormally, negative Poisson's ratio materials expand laterally when stretched and contract laterally when compressed. This enables them to offer enormous potential important applications [28], such as biomedicine [29] and protective equipments [30].

In recent years, negative Poisson's ratio effect has also been reported in several monolayer 2D materials. It is known that the out-of-plane and in-plane negative Poisson's ratios 
have been discovered in different black phosphorus [31-34]. The in-plane negative Poisson's ratio was also theoretically predicted in borophene [35], prismanes [36], transition metal dichalcogenides [37], and some not-yet-synthesized materials (e.g., penta-graphene) [38]. Recently, Grima's group [39] reported "how graphene can be modified to mimic the behavior of a highly and densely wrinkled paper model to the extent that it can exhibit negative Poission's $[s i c]$ ratio behavior under ambient conditions" for the first time. Significantly, Jiang and Park [40] theoretically explored the property of negative Poisson's ratio in single-layer graphene ribbons, which results from the compressive edge stressinduced warping of the edges. They claimed that "the effect is robust, as the negative Poission's [sic] ratio is observed for graphene ribbons with widths smaller than about 10 $\mathrm{nm}$, and for tensile strains smaller than about $0.5 \%$ with negative Poission's [sic] ratio values reaching as large as -1.51 ." Lately, they performed molecular dynamics (MD) simulations to investigate Poisson's ratio of graphene oxide and found that "the Poisson's ratio can be effectively tuned by increasing the degree of oxidation of graphene oxide." More specifically, "the Poisson's ratio decreases linearly from positive to negative with increasing of oxidation, and reaching a value of -0.567 for fully oxidized graphene" [41]. In addition, negative Poisson's ratio in rippled graphene due to the dewrinkling effect has been simulated by MD calculation [42].

Negative Poisson's ratio is theoretically possible within continuum elasticity for carbon nanostructures; it is often observed in engineered materials [43], as they are not intrinsic for many materials at the molecular level. Excitedly, also reported by Jiang's group [44], the single-layer graphene may exhibit an intrinsic negative Poisson's ratio, which is robust and independent of its size and temperature. They pointed out that "the negative Poission's [sic] ratio arises from the interplay between two intrinsic deformation pathways (one with positive Poisson's ratio, the other with negative Poission's [sic] ratio)." It is known that one of the major causes for a negative Poisson's ratio is the microstructure with a reentrant honeycomb geometric unit of the material at the molecular level $[45,46]$. Moreover, using the two-dimensional classical example of reentrant honeycomb [47], "it is clear that the auxetic effect would initially be large and would gradually decay as the dent in the hexagon flattens out." Accordingly, a pictorial representation of the system studied here and the auxetic structure designed to simulate are shown in Figure 1. This kind of topology geometry is expected to mimic the reentrant hexagonal honeycombs, which can be successfully created by the appropriate combination of $s p^{2}$ (phenyl) and $s p$ (alkynyl) carbon atoms like in graphyne. This will be further illustrated thereinafter. In this paper, we design a novel graphyne-like twodimensional carbon nanostructure with a reentrant hexagonal structural unit optimized by means of density functional theory and then try to predict its in-plane negative Poisson's ratio and other mechanical properties. We hope our work could offer a new strategy for the theoretical development of the $2 \mathrm{D}$ carbon nanostructure with the intrinsic negative Poisson's ratio.

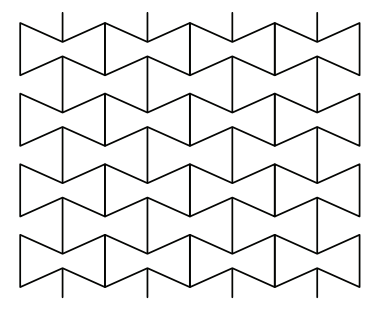

Figure 1: Pictorial representation of the reentrant hexagonal honeycombs.

\section{Computational Method}

All calculations, including the self-consistent energy calculations and structure optimization, were performed using the density functional theory of Perdew-Burke-Ernzerhof (PBE) exchange correlation with a generalized gradient approximation (GGA) functional [48] along with the projector-augmented wave (PAW) potentials $[49,50]$ as implemented in the Vienna Ab Initio Simulation Package (VASP) [51, 52]. An energy cutoff of $500 \mathrm{eV}$ was used. The monolayer structure is modelled in an orthorhombic cell that contains one formula unit (Figure 2) and a $20 \AA$ vacuum space inserted in the out-of-plane direction. A 3 $\times 5 \times 1$ k-point grid was used to sample the Brillouin zone during structure relaxation. All atoms were fully relaxed until their atomic forces were $<0.005 \mathrm{eV}^{-1}$. The effects of spin-orbit coupling on the structural deformation are considered to be minor and hence not included in our study. The vibration analyses for the relaxed structures were performed to confirm that these structures were thermodynamically stable.

A classical trajectory calculation using the atom-centered density matrix propagation ab initio molecular dynamics (MD) model [53-55], which can provide an equivalent functionality to Born-Oppenheimer molecular dynamics $[56,57]$ at considerably reduced computational cost, was performed for the preoptimized basic structural unit (neutral molecule) with different sizes so as to further infer and investigate the dynamic stabilities of the designed 2D structures.

Poisson's ratio is calculated from the engineering $\operatorname{strain}(\varepsilon)$, which is defined as the change in length $\Delta L$ per unit of the original length $L$, that is, $\varepsilon=\Delta L / L$. The applied uniaxial strain is realized in our calculations by fixing the lattice parameter to a value different from its equilibrium value during structural relaxation. The resulting strain in the transverse direction is extracted from the fully relaxed structure subjected to an applied strain.

\section{Results and Discussions}

The chemical structure of the designed graphyne-like 2D carbon nanomaterial is shown in Figure 3. It has a reentrant hexagon structural unit in which $m, n$, and $l$ correspond to the number of alkynyl at three different positions between phenyl units. It is found that when $m=1, n=1$, and $l=2$ (denoted as GL-2D-1, similar to Figure 2), the corresponding 2D structure is stable in thermodynamics; when $m=1, n=2$ and $l=2$, the corresponding $2 \mathrm{D}$ structure is also stable in 


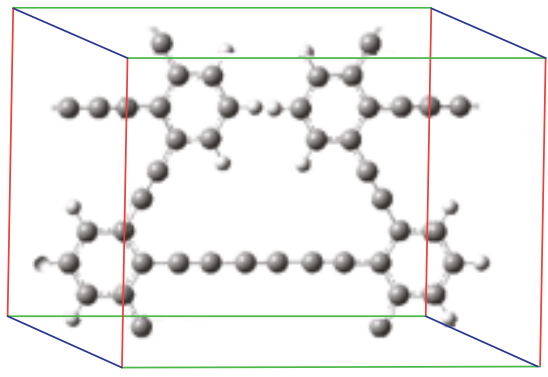

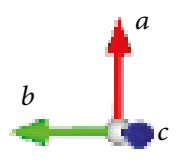

(a)

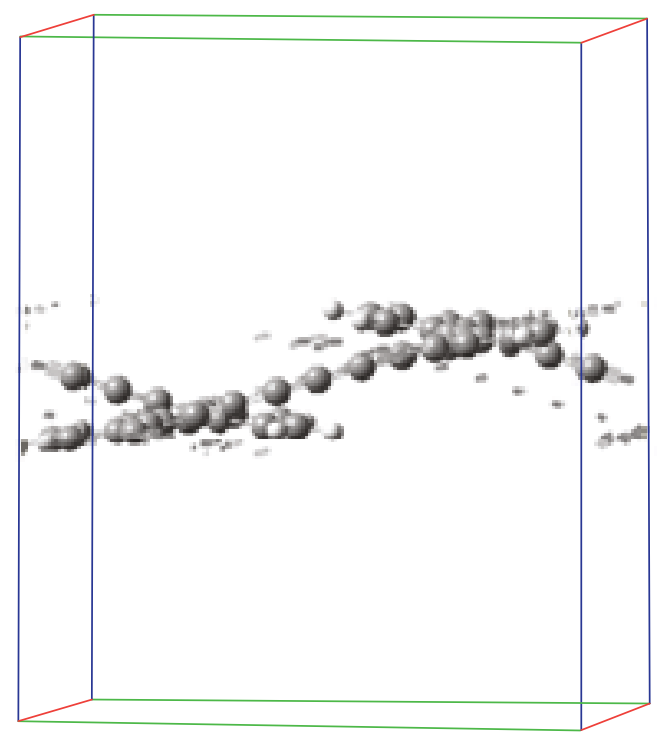

(b)

FIgURE 2: The designed periodical 2D carbon nanostructure (a) top view and (b) side view (carbon atoms in grey and hydrogen atoms in white).

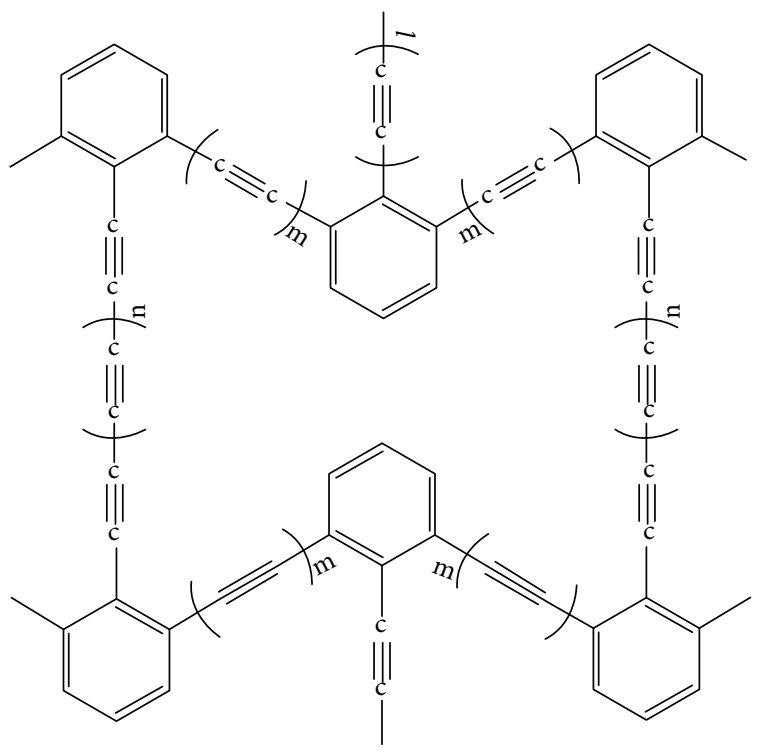

Figure 3: The chemical structure of the present designed graphdiyne-like $2 \mathrm{D}$ carbon nanomaterial $(m=1, n=1$, and $l=2)$.

thermodynamics, while when $m=1, n=2$ and $l=3$, the thermodynamic stable 2D structure cannot be obtained theoretically. Therefore, it can be seen that the length of the alkyne-linking chain is very important for the design of a reasonable 2D structure. Namely, it is not that the alkyne-linking chain with reasonable geometric length can get the corresponding stable 2D structure. Presently, the following investigations and discussions about mechanic properties are based on the structure of GL-2D-1 and its configurational isomer.

Figure 4 plots the configuration of the supercell of GL$2 \mathrm{D}-1$ at three orthogonal directions of $a, b, c$ and standard orientation together with the optimized lattice parameters of the unit cell. GL-2D-1 is a typical anisotropic 2D material, and it would have obvious differences in mechanical properties at $a$ and $b$ directions. Viewed from a direction, it is "butterfly"-shaped (Figure 4(a)).

In order to investigate the thermodynamic stabilities of the structures of GL-2D-1 and GL-2D-2, frequency analysis was performed at the same theoretical level to the structure optimizations. The results showed that there is no imaginary frequency for the two periodical 2D structures, indicating that these structures were thermodynamically stable on the potential energy surfaces. The data of frequency of the two 2D structures are listed in Table S1 and Table S2 of the Supporting Information.

Molecular dynamic stability is also as important as thermodynamic stability. Herein, ab initio MD was conducted for the preoptimized minimum basic structural unit (Figure S1) and hexamer of the basic structural unit (Figure S2) of GL-2D at room temperature, respectively, to evaluate the dynamic stabilities of the two designed 2D structures. Figure 5 shows the molecular dynamic simulations of the preoptimized minimum basic structural units of GL-2D-1 and GL-2D-2. It is clear that the largest changes in potential energies for the two structural units are only 5.78 and $4.98 \mathrm{~kJ} \cdot \mathrm{mol}^{-1}$ within $5000 \mathrm{fs}$, respectively, which are small enough to indicate the high molecular dynamic stabilities. Moreover, GL-2D-2 shows a slight higher molecular dynamic stability than does GL-2D-1 at 298 K. For completeness, the molecular dynamic simulations of the hexamer of the basic structural unit of GL-2D-1 and GL-2D-2 are shown in Figure S3 of the Supporting Information, and the largest changes in potential energies for the two hexamers are both smaller than $4.50 \mathrm{~kJ} \cdot \mathrm{mol}^{-1}(4.46$ and $4.27 \mathrm{~kJ} \cdot \mathrm{mol}^{-1}$ ) within $1000 \mathrm{fs}$, respectively, also indicating the excellent dynamic stabilities of the two $2 \mathrm{D}$ structures. 


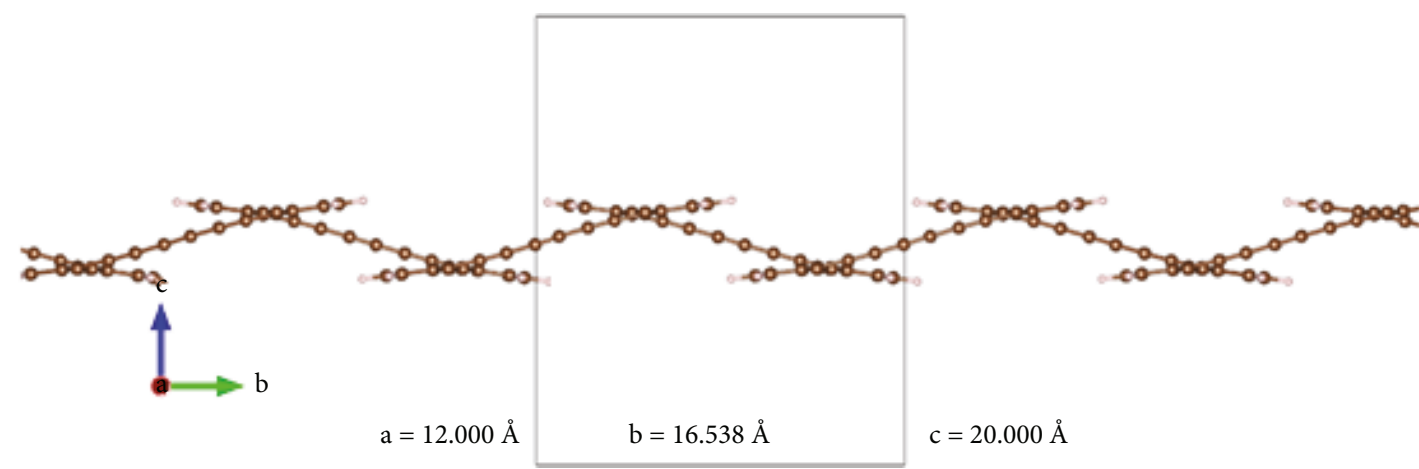

(a)

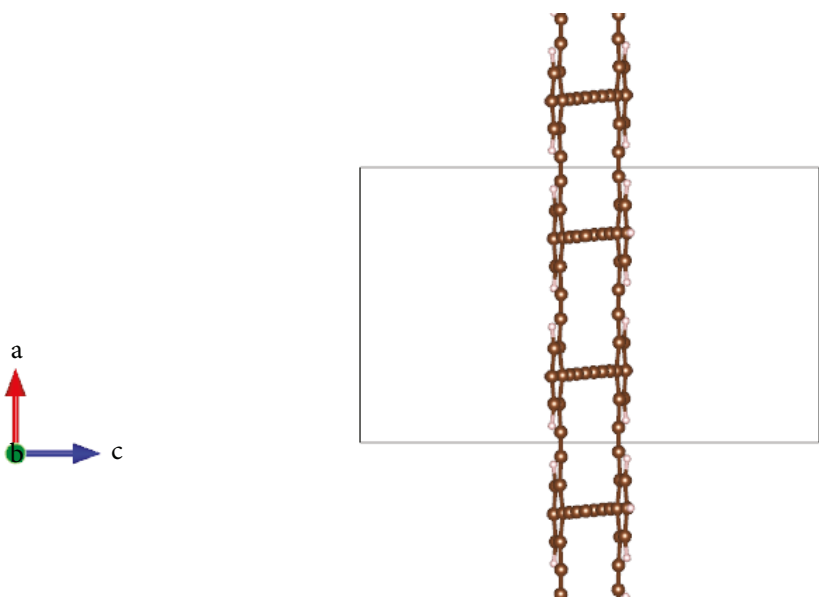

(b)

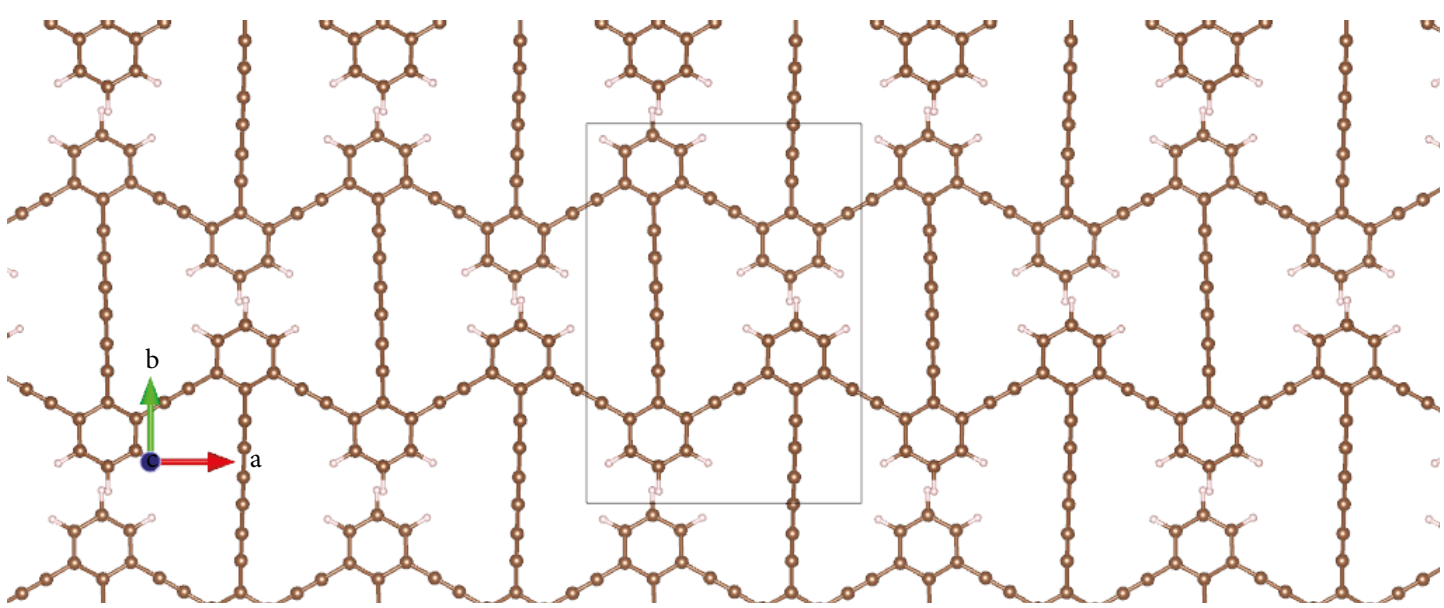

(c)

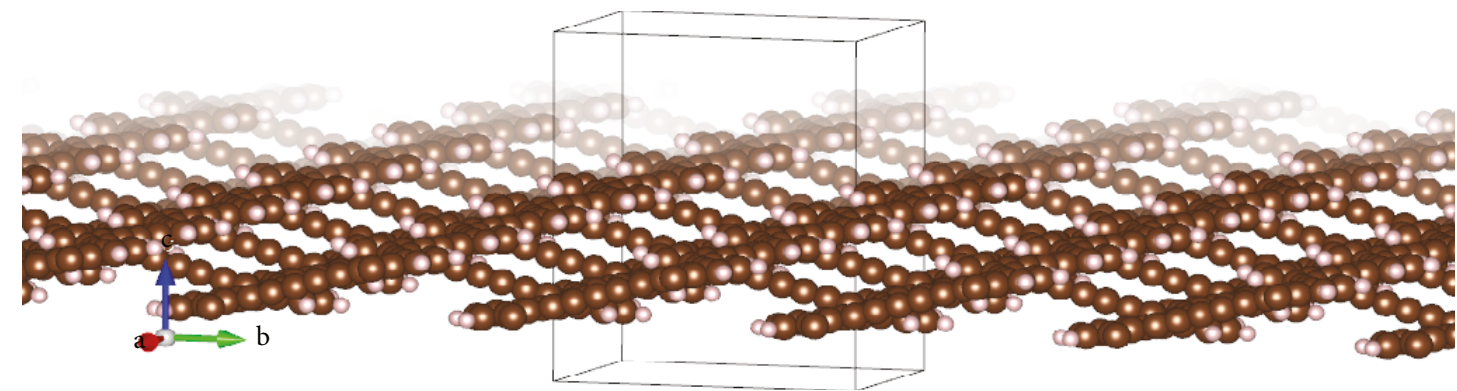

(d)

Figure 4: The configuration of the supercell of GL-2D-1 at three orthogonal directions of (a), (b), (c) and standard orientation (d). 


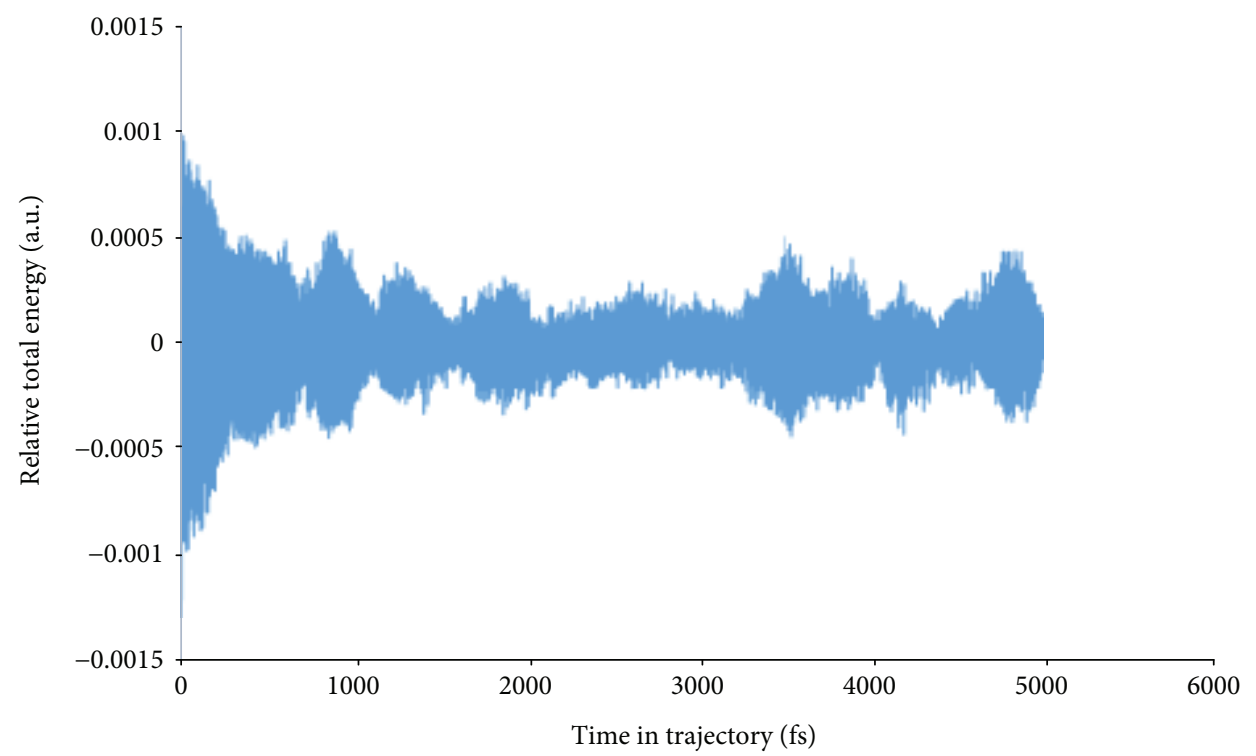

(a)

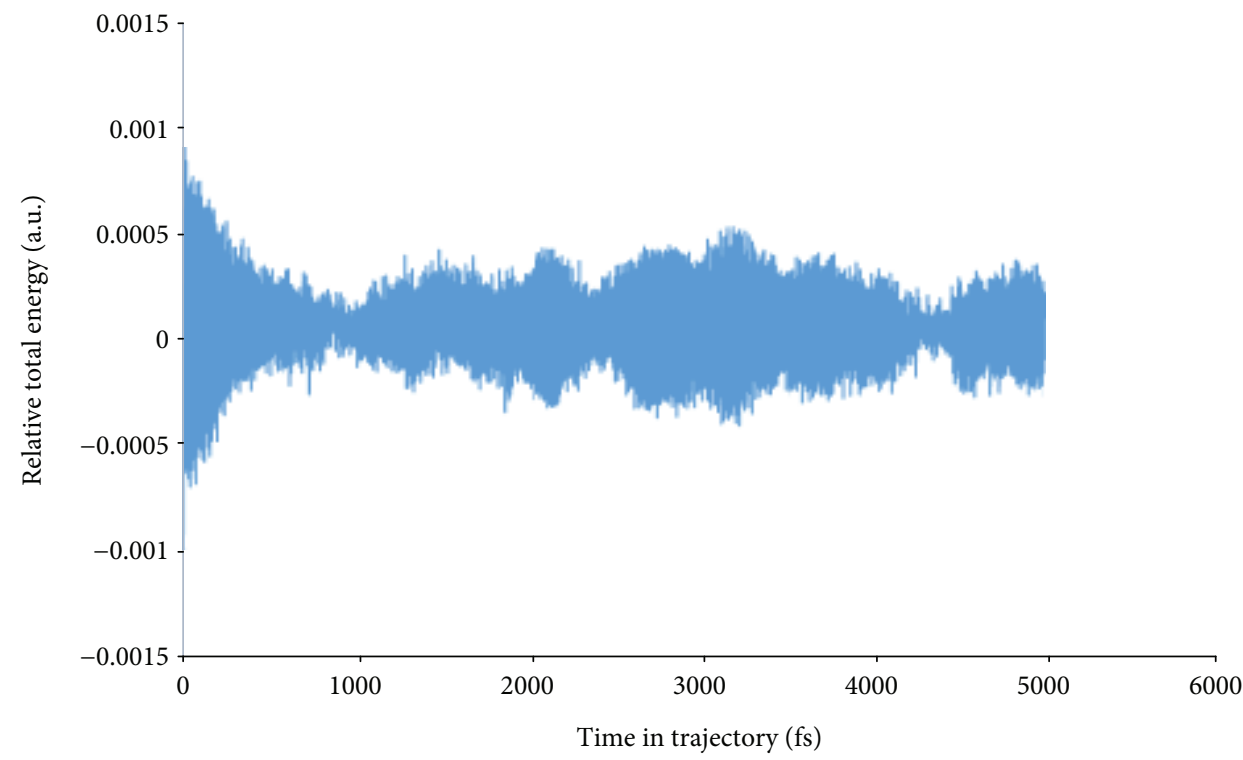

(b)

FIGURE 5: Molecular dynamic simulations of the preoptimized minimum basic structural units of (a) GL-2D-1 and (b) GL-2D-2.

Poisson's ratio is the most important mechanical property we concerned. Figures $6(\mathrm{a})$ and $6(\mathrm{~b})$ are Poisson's ratio of GL-2D-1 vs strain in $a$ and $b$ directions, respectively. It is clear that there is no negative Poisson's ratio effect when compressing along the $a$ direction. However, when stretching along the $a$ direction, the negative Poisson's ratio effect of GL-2D-1 is significant (Figure 6(a)). At the initial stage of stretching, the material has a very large negative Poisson's ratio; with the increase in the tensile strain, the negative Poisson ratio effect is weakened, and the Poisson's ratio $v$ converges to a certain value. As Figure 6(b) shows, the negative Poisson's ratio effect in the $b$ direction is contrary to that of the $a$ direction. There is no negative Poisson's ratio effect when stretching along the $b$ direction, while when compressing along the $b$ direction, the negative Poisson's ratio effect of GL-2D-1 is obvious, although it is weaker than that in the case of stretching along the $a$ direction. Similarly, with the increase in the compression strain in the $b$ direction, the negative Poisson ratio effect is weakened, and then the Poisson's ratio $v$ converges to a certain value. In brief, GL-2D-1 can present the significant negative Poisson ratio effect both in $a$ and $b$ directions under different specific strains.

Additionally, other mechanical properties were further investigated. The stiffness matrix and its six eigenvalues are given in Table S3 and Table S4 of the Supporting Information. Seen from the stiffness matrix of GL-2D-1, it is not a positive definite matrix, manifesting that the mechanical stability of the designed GL-2D-1 is not as excellent as that of graphdiyne. However, it is noted that among the six eigenvalues $\left(\lambda_{1} \sim \lambda_{6}\right), \lambda_{1} \sim \lambda_{4}$ are negative 


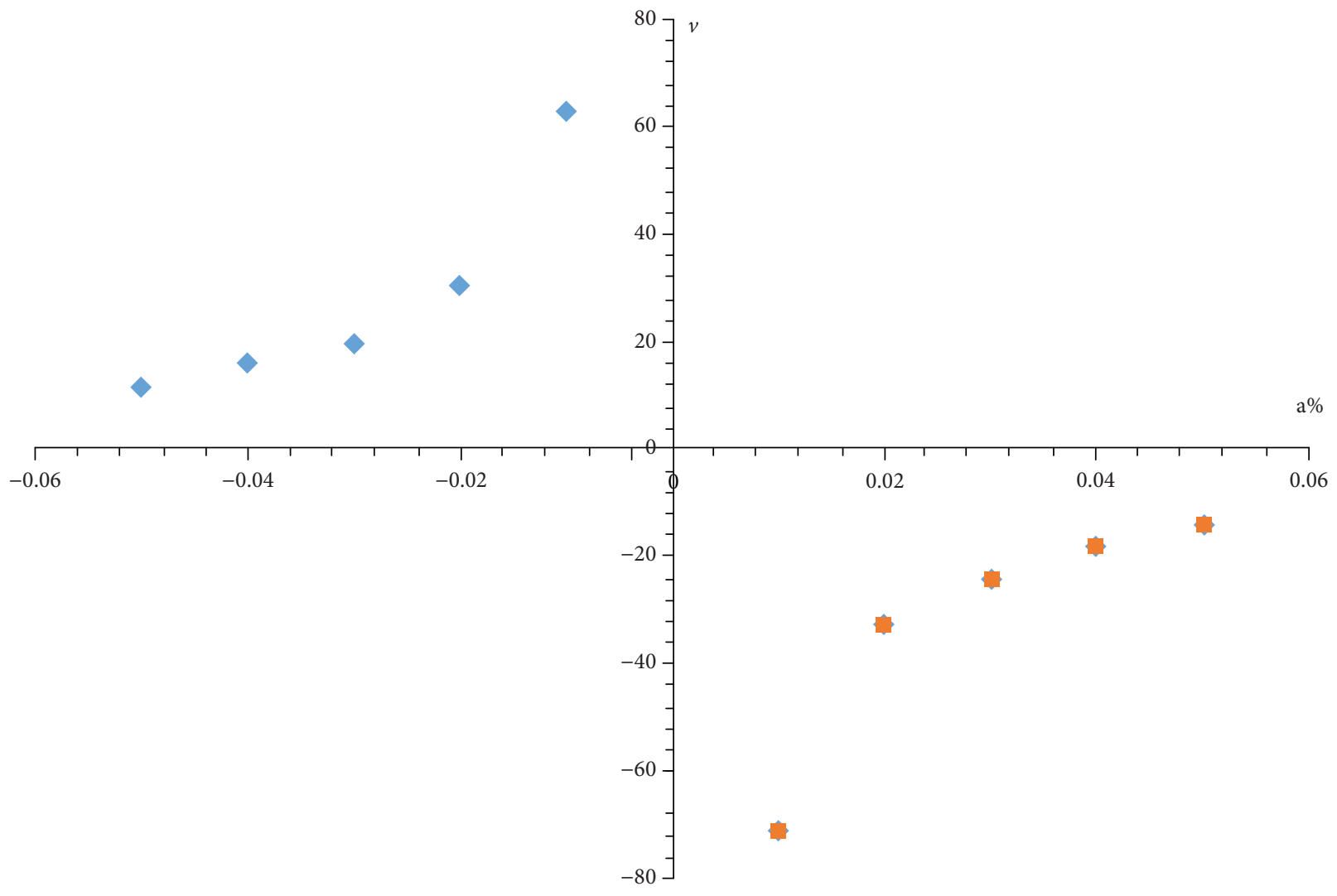

(a)

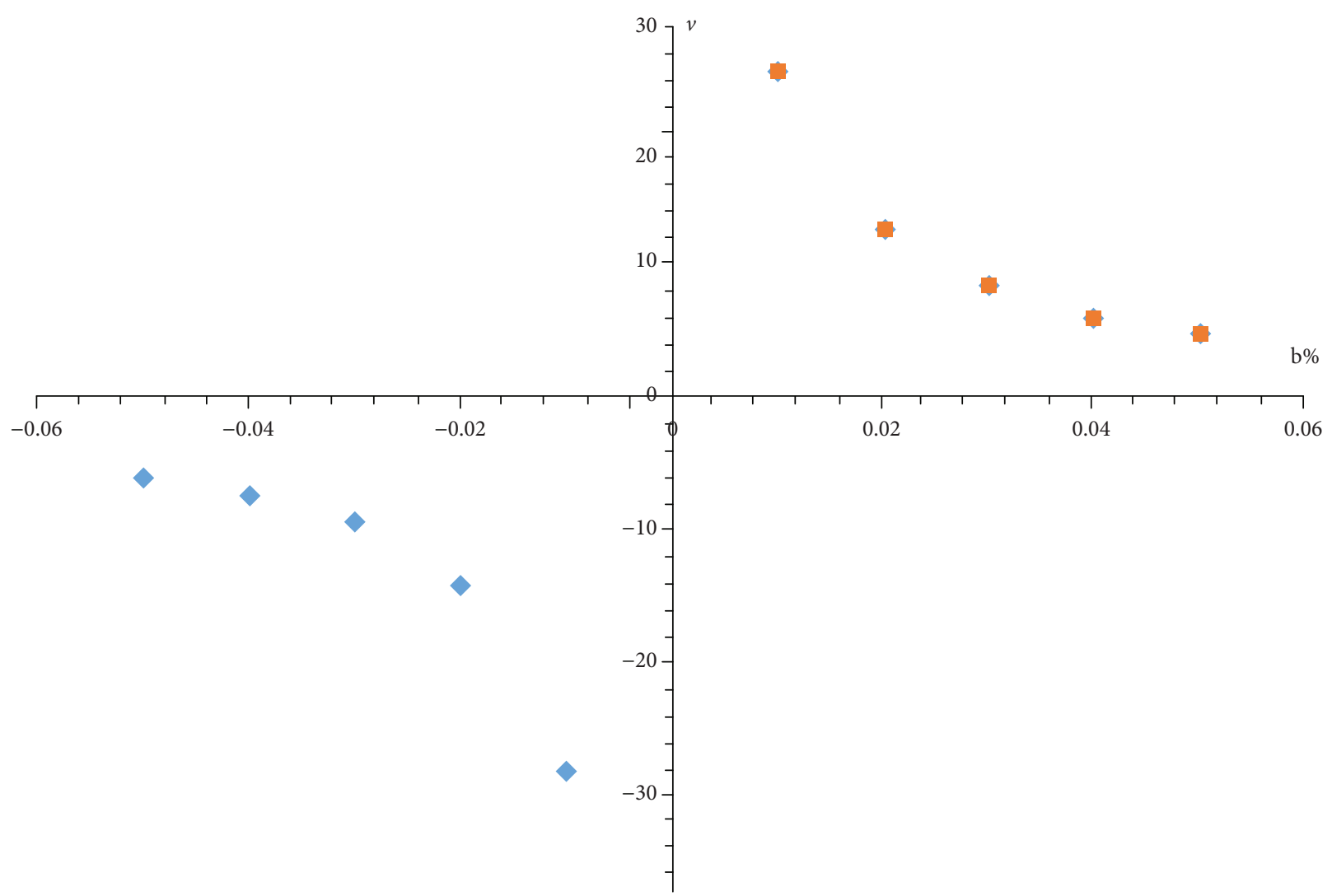

(b)

FIgUre 6: Poisson's ratio of GL-2D-1 vs strain in the $a$ direction (a) and $b$ direction (b). 


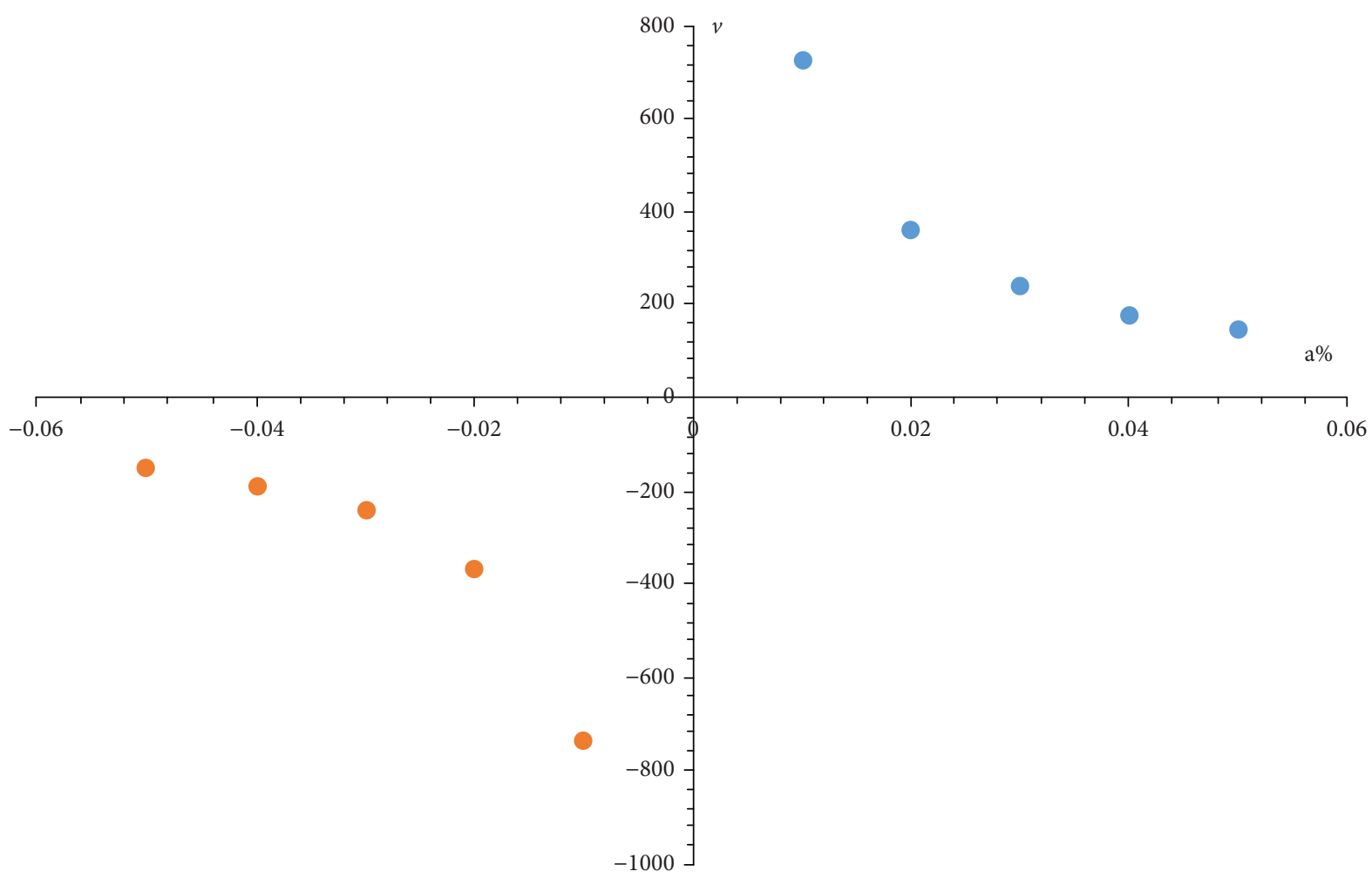

(a)

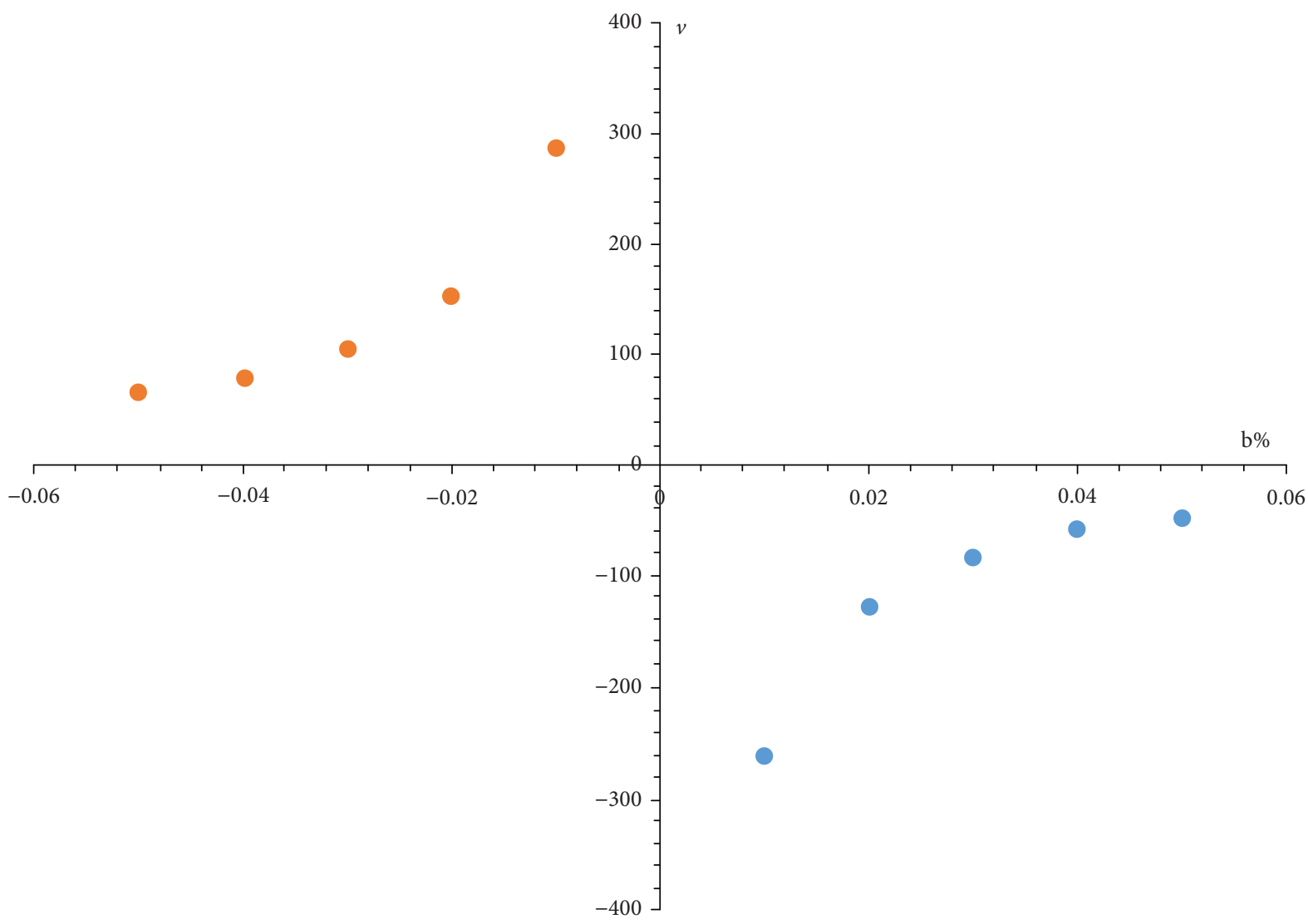

(b)

FIGURE 7: Poisson's ratio of GL-2D-2 vs strain in the $a$ direction (a) and $b$ direction (b). 
with a smaller absolute value, and $\lambda_{5}$ and $\lambda_{6}$ are positive, indicating that GL-2D-1 has a certain mechanical stability. Moreover, the modulus of GL-2D-1 under different models including Voigt, Reuss, and Hill are also presented in Table S5 of the Supporting Information.

Interestingly, a configuration isomer of GL-2D-1 is found, denoted as GL-2D-2, which is also a thermodynamically stable 2D structure. Figure S4 of the Supporting Information shows the configuration of the supercell of GL-2D-2 at three orthogonal directions of $a, b, c$ and standard orientation. Viewed from a direction, it is a "herring-bone" form (Figure S4(a)). Compared to GL-2D1 , the negative Poisson's ratio effect of GL-2D-2 is exactly opposite. Figure 7 shows the Poisson's ratio of GL-2D-2 vs strain in the $a$ direction and $b$ direction. It can be seen that GL-2D-2 presents the negative Poisson's ratio effect when compressing along the $a$ direction or stretching along the $b$ direction and the normal Poisson's ratio effect when stretching along the $a$ direction or compressing along the $b$ direction. Either in the initial stage of stretching or in that of compressing, the absolute values of Poisson's ratio of GL-2D-2 are very large, but with the increasing of strains, they gradually decrease and converge to a certain value. Poisson's ratio has a "hyperbolic" rule with the whole trend of strain variation.

It is particularly noteworthy that although the negative Poisson's ratio effect of GL-2D-1 under certain strain conditions is significant, those of GL-2D-2 under the compression along the $a$ direction or stretching along the $b$ direction are distinctly larger than those of GL-2D-1 under stretching along the $a$ direction or compression along the $b$ direction. For example, Poisson's ratio of GL-2D-2 is -730.37 when it is compressed by $0.01 \%$ along the $a$ direction, while at the same stretching value along the same direction, that of GL-2D-1 is only -63.34 . Thereby, it can be found that the small differences in configuration may bring very large differences in the negative Poisson's ratio effect of the present 2D structures. This is of great significance for designing and recognizing 2D carbon nanostructures with negative Poisson's ratio effect.

For comparison, the stiffness matrix and its six eigenvalues and the modulus of GL-2D-2 are also given in Table S6, Table S7, and Table S8 of the Supporting Information. Similar to that of GL-2D-1, the stiffness matrix of GL-2D-2 is not a positive definite matrix, meaning that the mechanical stability is not very ideal. However, we noted that among the six eigenvalues of the stiffness matrix, $\lambda_{3} \sim \lambda_{6}$ are all positive values, and $\lambda_{1}$ and $\lambda_{2}$ are negative with very small absolute values, indicating that the mechanical stability of GL-2D-2 increased obviously compared to that of GL-2D-1.

\section{Conclusion}

In summary, a novel thermodynamic stable graphyne-like two-dimensional carbon nanostructure (GL-2D-1) with in-plane negative Poisson's ratio is designed and predicted by means of density functional theory and PBE exchange correlation with a GGA functional along with the PAW potentials. Simultaneously, an unforeseen stable conformational isomer of GL-2D-1, GL-2D-2, is also found theoretically. Excitedly, both GL-2D-1 and GL-2D-2 all present a significant negative Poisson's ratio effect under different specific strain conditions. Additionally, it is found that the small differences in conformation may bring very large differences in the negative Poisson's ratio effect of the 2D materials with the same configuration. We hope that our work could offer a new strategy for the theoretical development of the 2D carbon nanostructure with the intrinsic negative Poisson's ratio.

\section{Data Availability}

The data used to support the findings of this study are included within the article and Supplementary materials.

\section{Conflicts of Interest}

The authors declare that there is no conflict of interest regarding the publication of this paper.

\section{Acknowledgments}

This project was funded by the National Natural Science Foundation of China (No. 21663024 and 21663025), the China Postdoctoral Science Foundation (No. 2016M60 2809), the "Feitian" Young Scholar Program, Longyuan Youth Talent Innovative Support Program of Gansu Province, and the Scientific Research Project in Gansu Universities (2018A-076).

\section{Supplementary Materials}

Figure S1: the preoptimized minimum basic structural unit. Figure S2: the preoptimized hexamer of the basic structural unit. Table S1: the frequency analysis result for the periodical structure of GL-2D-1. Table S2: the frequency analysis result for the periodical structure of GL-2D-2. Figure S3: the molecular dynamic simulations of the hexamer of the basic structural unit of GL-2D-1 and GL-2D-2. Table S3: stiffness matrix (coefficients in GPa) of GL-2D-1. Table S4: eigenvalue of the stiffness matrix of GL-2D-1. Table S5: modulus of GL-2D-1 under different models. Table S6: stiffness matrix (coefficients in GPa) of GL-2D-2. Table S7: eigenvalue of stiffness matrix of GL-2D-2. Table S8: modulus of GL-2D-2 under a different model. Figure S4: the configuration of the supercell of GL-2D-2 at three orthogonal directions of $a, b$, $c$ and standard orientation. (Supplementary Materials)

\section{References}

[1] D. Umadevi, S. Panigrahi, and G. N. Sastry, "Noncovalent interaction of carbon nanostructures," Accounts of Chemical Research, vol. 47, no. 8, pp. 2574-2581, 2014.

[2] V. V. Danilenko, "On the history of the discovery of nanodiamond synthesis," Physics of the Solid State, vol. 46, no. 4, pp. 595-599, 2004.

[3] A. Hirsch, "The era of carbon allotropes," Nature Materials, vol. 9, no. 11, pp. 868-871, 2010.

[4] S. Iijima, "Helical microtubules of graphitic carbon," Nature, vol. 354 , no. 6348, pp. 56-58, 1991. 
[5] C. N. R. Rao, A. K. Sood, K. S. Subrahmanyam, and A. Govindaraj, "Graphene: the new two-dimensional nanomaterial," Angewandte Chemie International Edition, vol. 48, no. 42, pp. 7752-7777, 2009.

[6] S. E. Lewis, "Cycloparaphenylenes and related nanohoops," Chemical Society Reviews, vol. 44, no. 8, pp. 2221-2304, 2015.

[7] G. Li, Y. Li, H. Liu, Y. Guo, Y. Li, and D. Zhu, "Architecture of graphdiyne nanoscale films," Chemical Communications, vol. 46, no. 19, pp. 3256-3258, 2010.

[8] X. L. Sheng, Q. B. Yan, F. Ye, Q. R. Zheng, and G. Su, "T-Carbon: a novel carbon allotrope," Physical Review Letters, vol. 106, no. 15, article 155703, 2011.

[9] J. Zhang, R. Wang, X. Zhu et al., "Pseudo-topotactic conversion of carbon nanotubes to T-carbon nanowires under picosecond laser irradiation in methanol," Nature Communications, vol. 8, no. 1, p. 683, 2017.

[10] J. O. Sofo, A. S. Chaudhari, and G. D. Barber, "Graphane: a two-dimensional hydrocarbon," Physical Review B, vol. 75, no. 15, article 153401, 2007.

[11] C. Zhou, S. Chen, J. Lou et al., "Graphene's cousin: the present and future of graphane," Nanoscale Research Letters, vol. 9, no. 1, pp. 26-26, 2014.

[12] D. C. Elias, R. R. Nair, T. M. G. Mohiuddin et al., "Control of graphene's properties by reversible hydrogenation: evidence for graphane," Science, vol. 323, no. 5914, pp. 610613, 2009.

[13] S. Y. Ma, L. Z. Sun, and K. W. Zhang, "Prediction of two planar carbon allotropes with large meshes," Physical Chemistry Chemical Physics, vol. 18, no. 2, pp. 1172-1177, 2016.

[14] J. T. Wang, C. Chen, H. Mizuseki, and Y. Kawazoe, "New carbon allotropes in $\mathrm{sp}+\mathrm{sp}^{3}$ bonding networks consisting of $\mathrm{C}_{8}$ cubes," Physical Chemistry Chemical Physics, vol. 20, no. 12, pp. 7962-7967, 2018.

[15] P. Hobza, "Calculations on noncovalent interactions and databases of benchmark interaction energies," Accounts of Chemical Research, vol. 45, no. 4, pp. 663-672, 2012.

[16] G. Eda and M. Chhowalla, "Chemically derived graphene oxide: towards large-area thin-film electronics and optoelectronics," Advanced Materials, vol. 22, no. 22, pp. 2392-2415, 2010.

[17] J. Chen, J. Xi, D. Wang, and Z. Shuai, "Carrier mobility in graphyne should be even larger than that in graphene: a theoretical prediction," The Journal of Physical Chemistry Letters, vol. 4, no. 9, pp. 1443-1448, 2013.

[18] H. Tang, C. M. Hessel, J. Wang et al., "Two-dimensional carbon leading to new photoconversion processes," Chemical Society Reviews, vol. 43, no. 13, pp. 4281-4299, 2014.

[19] A. K. Geim and I. V. Grigorieva, "Van der Waals heterostructures," Nature, vol. 499, no. 7459, pp. 419-425, 2013.

[20] P. Miro, M. Audiffred, and T. Heine, "An atlas of twodimensional materials," Chemical Society Reviews, vol. 43, no. 18, pp. 6537-6554, 2014.

[21] M.-M. Titirici, R. J. White, N. Brun et al., "Sustainable carbon materials," Chemical Society Reviews, vol. 44, no. 1, pp. 250290, 2015.

[22] X. N. Sun, D. Xu, W. Hu, and X. Y. Chen, "Template synthesis of 2D carbon nanosheets: improving energy density of supercapacitors by dual redox additives anthraquinone-2-sulfonic acid sodium and KI," ACS Sustainable Chemistry \& Engineering, vol. 5, no. 7, pp. 5972-5981, 2017.
[23] Z. Jia, Y. Li, Z. Zuo, H. Liu, C. Huang, and Y. Li, "Synthesis and properties of 2D carbon-graphdiyne," Accounts of Chemical Research, vol. 50, no. 10, pp. 2470-2478, 2017.

[24] Y. Ding, Q. Chang, F. Xiu et al., "Zero- and two-dimensional hybrid carbon phosphors for high colorimetric purity white light-emission," Nanoscale, vol. 10, no. 9, pp. 4189-4193, 2018.

[25] L.-H. Zhang, W.-C. Li, H. Liu et al., "Thermoregulated phasetransition synthesis of two-dimensional carbon nanoplates rich in $\mathrm{sp}^{2}$ carbon and unimodal ultramicropores for kinetic gas separation," Angewandte Chemie International Edition, vol. 57, no. 6, pp. 1632-1635, 2018.

[26] X. Li and L. Zhi, "Graphene hybridization for energy storage applications," Chemical Society Reviews, vol. 47, no. 9, pp. 3189-3216, 2018.

[27] R. Lakes, "Foam structures with a negative Poisson's ratio," Science, vol. 235, no. 4792, pp. 1038-1040, 1987.

[28] G. N. Greaves, A. L. Greer, R. S. Lakes, and T. Rouxel, "Poisson's ratio and modern materials," Nature Materials, vol. 10, no. 11, pp. 823-837, 2011.

[29] F. Scarpa, "Auxetic materials for bioprostheses [In the Spotlight]," IEEE Signal Processing Magazine, vol. 25, no. 5, 2008.

[30] M. Sanami, N. Ravirala, K. Alderson, and A. Alderson, "Auxetic materials for sports applications," Procedia Engineering, vol. 72, pp. 453-458, 2014.

[31] J. W. Jiang and H. S. Park, "Negative Poisson's ratio in singlelayer black phosphorus," Nature Communications, vol. 5, no. 1, p. 4727, 2014.

[32] J. W. Jiang, T. Rabczuk, and H. S. Park, "A Stillinger-Weber potential for single-layered black phosphorus, and the importance of cross-pucker interactions for a negative Poisson's ratio and edge stress-induced bending," Nanoscale, vol. 7, no. 14, pp. 6059-6068, 2015.

[33] Y. Du, J. Maassen, W. Wu, Z. Luo, X. Xu, and P. D. Ye, “Auxetic black phosphorus: a 2D material with negative Poisson's ratio," Nano Letters, vol. 16, no. 10, pp. 6701-6708, 2016.

[34] D. T. Ho, V. H. Ho, H. S. Park, and S. Y. Kim, "Negative inplane Poisson's ratio for single layer black phosphorus: an atomistic simulation study," Physica Status Solidi (B), vol. 254, no. 12, article 1700285, 2017.

[35] A. J. Mannix, X.-F. Zhou, B. Kiraly et al., "Synthesis of borophenes: anisotropic, two-dimensional boron polymorphs," Science, vol. 350, no. 6267, pp. 1513-1516, 2015.

[36] N. Pour, E. Altus, H. Basch, and S. Hoz, "The origin of the auxetic effect in prismanes: bowtie structure and the mechanical properties of biprismanes," The Journal of Physical Chemistry C, vol. 113, no. 9, pp. 3467-3470, 2009.

[37] L. Yu, Q. Yan, and A. Ruzsinszky, “Negative Poisson's ratio in 1T-type crystalline two-dimensional transition metal dichalcogenides," Nature Communications, vol. 8, article 15224, 2017.

[38] S. Zhang, J. Zhou, Q. Wang, X. Chen, Y. Kawazoe, and P. Jena, "Penta-graphene: a new carbon allotrope," Proceedings of the National Academy of Sciences of the United States of America, vol. 112, no. 8, pp. 2372-2377, 2015.

[39] J. N. Grima, S. Winczewski, L. Mizzi et al., "Tailoring graphene to achieve negative Poisson's ratio properties," Advanced Materials, vol. 27, no. 8, pp. 1455-1459, 2015.

[40] J. W. Jiang and H. S. Park, "Negative Poisson's ratio in singlelayer graphene ribbons," Nano Letters, vol. 16, no. 4, pp. 2657$2662,2016$. 
[41] J. Wan, J. W. Jiang, and H. S. Park, “Negative Poisson's ratio in graphene oxide," Nanoscale, vol. 9, no. 11, pp. 4007-4012, 2017.

[42] H. Qin, Y. Sun, J. Z. Liu, M. Li, and Y. Liu, "Negative Poisson's ratio in rippled graphene," Nanoscale, vol. 9, no. 12, pp. 41354142, 2017.

[43] C. Huang and L. Chen, "Negative Poisson's ratio in modern functional materials," Advanced Materials, vol. 28, no. 37, pp. 8079-8096, 2016.

[44] J. W. Jiang, T. Chang, X. Guo, and H. S. Park, "Intrinsic negative Poisson's ratio for single-layer graphene," Nano Letters, vol. 16, no. 8, pp. 5286-5290, 2016.

[45] N. Pour, L. Itzhaki, B. Hoz, E. Altus, H. Basch, and S. Hoz, "Auxetics at the molecular level: a negative Poisson's ratio in molecular rods," Angewandte Chemie International Edition, vol. 45, no. 36, pp. 5981-5983, 2006.

[46] L. Mizzi, K. M. Azzopardi, D. Attard, J. N. Grima, and R. Gatt, "Auxetic metamaterials exhibiting giant negative Poisson's ratios," Physica Status Solidi (RRL) - Rapid Research Letters, vol. 9, no. 7, pp. 425-430, 2015.

[47] K. E. Evans, M. A. Nkansah, I. J. Hutchinson, and S. C. Rogers, "Molecular network design," Nature, vol. 353, no. 6340, p. 124, 1991.

[48] J. P. Perdew, K. Burke, and M. Ernzerhof, "Generalized gradient approximation made simple," Physical Review Letters, vol. 77, no. 18, pp. 3865-3868, 1996.

[49] P. E. Blochl, "Projector augmented-wave method," Physical Review B, vol. 50, no. 24, pp. 17953-17979, 1994.

[50] G. Kresse and D. Joubert, "From ultrasoft pseudopotentials to the projector augmented-wave method," Physical Review B, vol. 59, no. 3, pp. 1758-1775, 1999.

[51] G. Kresse and J. Furthmüller, "Efficient iterative schemes for $\mathrm{ab}$ initio total-energy calculations using a plane-wave basis set," Physical Review B, vol. 54, no. 16, pp. 11169-11186, 1996.

[52] G. Kresse and J. Furthmüller, "Efficiency of ab-initio total energy calculations for metals and semiconductors using a plane-wave basis set," Computational Materials Science, vol. 6, no. 1, pp. 15-50, 1996.

[53] S. S. Iyengar, H. B. Schlegel, J. M. Millam, G. A. Voth, G. E. Scuseria, and M. J. Frisch, "Ab initio molecular dynamics: propagating the density matrix with Gaussian orbitals. II. Generalizations based on mass-weighting, idempotency, energy conservation and choice of initial conditions," The Journal of Chemical Physics, vol. 115, no. 22, pp. 10291-10302, 2001.

[54] H. B. Schlegel, J. M. Millam, S. S. Iyengar et al., "Ab initiomolecular dynamics: propagating the density matrix with Gaussian orbitals," The Journal of Chemical Physics, vol. 114, no. 22, pp. 9758-9763, 2001.

[55] H. B. Schlegel, S. S. Iyengar, X. Li et al., "Ab initio molecular dynamics: propagating the density matrix with Gaussian orbitals. III. Comparison with Born-Oppenheimer dynamics," The Journal of Chemical Physics, vol. 117, no. 19, pp. 86948704, 2002.

[56] T. Helgaker, E. Uggerud, and H. J. A. Jensen, "Integration of the classical equations of motion on ab initio molecular potential energy surfaces using gradients and Hessians: application to translational energy release upon fragmentation," Chemical Physics Letters, vol. 173, no. 2-3, pp. 145-150, 1990.

[57] E. Uggerud and T. Helgaker, "Dynamics of the reaction $\mathrm{CH} 2 \mathrm{OH}+$.fwdarw. $\mathrm{CHO}++\mathrm{H} 2$. Translational energy release from ab initio trajectory calculations," Journal of the American Chemical Society, vol. 114, no. 11, pp. 4265-4268, 1992. 


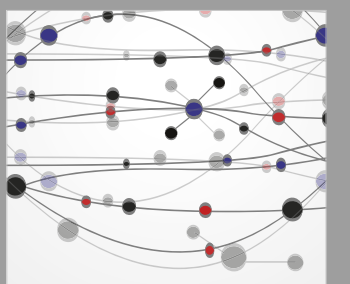

The Scientific World Journal
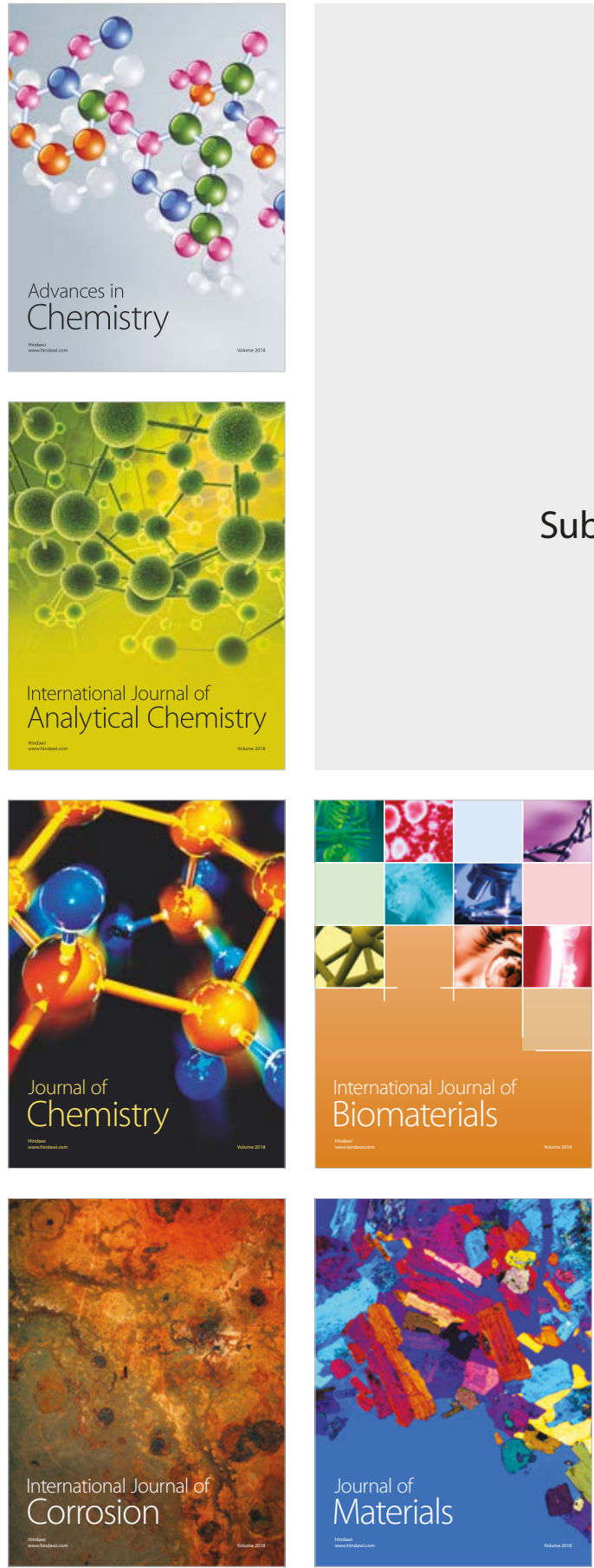

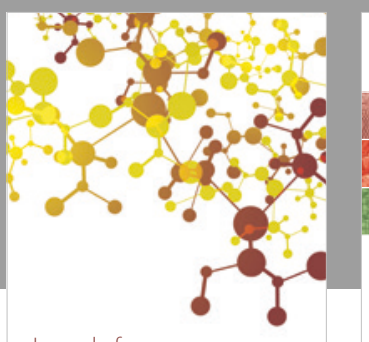

Journal of

Applied Chemistry
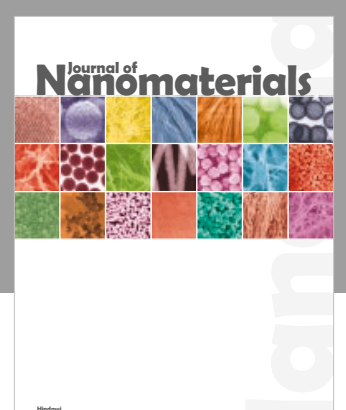

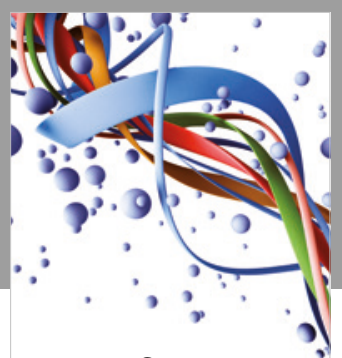

Scientifica

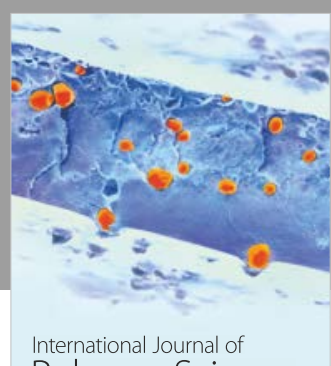

Polymer Science

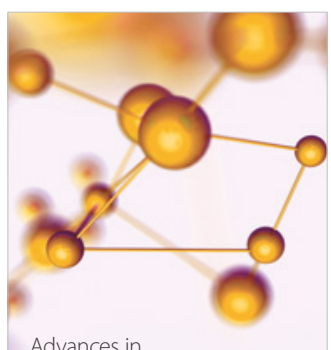

Physical Chemistry
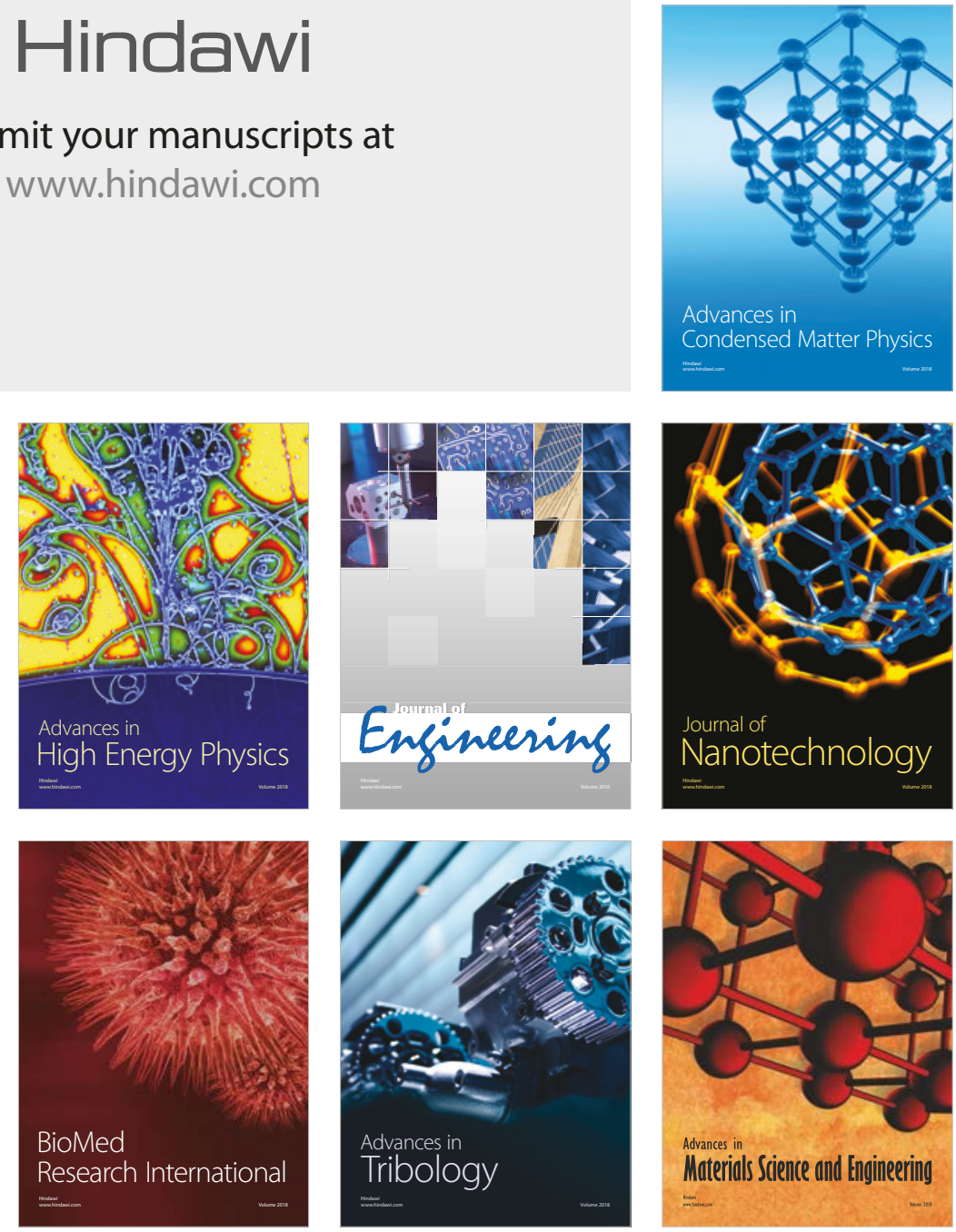